\title{
The Association Between Nailfold Capillary Density and Pulmonary and Cardiac Involvement in Medium to Longstanding Juvenile Dermatomyositis
}

Zoltan Barth $\mathrm{MD}^{1,2,3}$, Thomas Schwartz MD, PhD1, Berit Flatø MD PhD ${ }^{4,5}$, Trond Mogens Aaløkken MD PhD ${ }^{6}$, Akos Koller MD, $\mathrm{PhD}^{7,8}$, May Brit Lund MD, $\mathrm{PhD}^{5,9}$, Ivar Sjaastad MD PhD ${ }^{1,10}$, Helga Sanner MD PhD ${ }^{3,4}$

${ }^{1}$ Institute for Experimental Medical Research and KG Jebsen Center for Cardiac Research, Oslo University Hospital-Ullevål and University of Oslo, Oslo, Norway ${ }^{2}$ Institute for Translational Medicine, Medical School, University of Pécs, Pécs, Hungary

${ }^{3}$ Bjørknes University College, Oslo, Norway

${ }^{4}$ Department of Rheumatology, Oslo University Hospital-Rikshospitalet, Oslo, Norway ${ }^{5}$ Institute for Clinical Medicine, Medical Faculty, University of Oslo, Oslo, Norway ${ }^{6}$ Department of Radiology, Oslo University Hospital-Rikshospitalet, Oslo, Norway ${ }^{7}$ Department of Neurosurgery, Medical School, University of Pécs, Hungary ${ }^{8}$ Institute of Natural Sciences, University of Physical Education, Budapest, Hungary ${ }^{9}$ Department of Respiratory Medicine, Oslo University Hospital-Rikshospitalet, Oslo, Norway

${ }^{10}$ Department of Cardiology, Oslo University Hospital-Ullevål, Oslo, Norway

Dr Sjaastad is supported by Center for Heart Failure Research, University of Oslo, Oslo, Norway, and Anders Jahres Fund for the promotion of science. Dr Koller is supported by National Research, Development and Innovation Office (OTKA K 
108444), Hungary. Dr Barth was supported by Campus Hungary Program (TÁMOP4.2.4B/2-11/1-2012-0001).

Competing interests: none.

Address correspondence to:

Helga Sanner, MD, PhD, Department of Rheumatology, Oslo University Hospital-

Rikshospitalet, 0027 Oslo, Norway

Telephone: 004723073323

Fax: 004723072990

E-mail: helga.sanner@medisin.uio.no 


\section{Abstract}

Objective. To explore the associations between microvascular abnormalities assessed by nailfold capillaroscopy (NFC) and pulmonary and cardiac involvement in patients with juvenile dermatomyositis (DM) assessed after medium- to long-term follow-up.

Methods. Fifty-eight juvenile DM patients were examined mean 17.0 (SD 10.6) years after symptom onset. Nailfold capillary density (NCD) and neovascular pattern (defined as scleroderma active or late pattern) were analysed blinded to clinical data. Pulmonary involvement was assessed by pulmonary function tests (PFT) including spirometry, diffusion capacity for carbon monoxide (DLCO) and body plethysmography; also high-resolution computed tomography (HRCT) was performed. Cardiac involvement was assessed by electrocardiography, Holter monitoring (heart rate variability) and echocardiography.

Results. Patients with low NCD ( $<6$ capillaries $/ \mathrm{mm})(\mathrm{n}=21)$, compared to patients with normal NCD ( $\geq 6$ capillaries $/ \mathrm{mm})(\mathrm{n}=37)$, presented lower forced vital capacity ( 89.7 vs $98.5 \%$ of predicted), total lung capacity ( 87.8 vs $94.5 \%$ of predicted) and more often low DLCO (15/21 (71\%) vs 14/37 (38\%)); all p's<0.05. HRCT assessed airways disease was more frequent in the low NCD group (6/21 (30\%) vs $3 / 37(8 \%)$; $p=0.034$ ). No associations were found between i) NCD and cardiac parameters or ii) between neovascular pattern and pulmonary or cardiac parameters.

Conclusion. In juvenile DM patients, low NCD was associated with lung involvement, which was mostly subclinical. No significant associations with cardiac involvement were found. These results shed light on possible mechanisms underlying 
NFC and organ Involvement in Juvenile DM

organ involvement, but further and preferably larger studies are needed to examine NCD as a potential biomarker for lung and cardiac involvement in juvenile DM. 


\section{Significance \& Innovations}

1. In juvenile dermatomyositis (DM) patients assessed after medium- to longterm follow-up, low nailfold capillary density (NCD) is associated with impaired pulmonary function tests and HRCT CT detected airways disease.

2. NCD is not significantly associated with cardiac involvement, including systolic and diastolic dysfunction, echocardiographic abnormalities and heart rate variability.

3. These findings shed light upon the role of microvascular remodelling for organ involvement, but further and larger studies are needed to investigate the possible role of NCD as a biomarker for organ involvement in juvenile DM. 


\section{Introduction}

Juvenile dermatomyositis (DM) is a rare autoimmune myopathy with childhood origin, characterized primarily by pathognomonic skin rashes and muscle weakness. Juvenile DM is considered to be a multi-systemic vasculopathy where autoimmune mechanisms target small vessels. The systemic vasculopathy and the consequent microvascular remodelling might play an important role in the involvement of various organs, including the heart and lungs.

Pulmonary involvement is a relatively infrequent complication in juvenile DM (1), associated with poor prognosis (1). Interstitial lung disease (ILD) and impaired pulmonary function tests (PFT) are the most frequent findings. Although pulmonary involvement is mostly subclinical, rapidly progressive ILD was found as a major cause of death in Japanese patients with juvenile DM (2). Clinically important cardiac involvement is even more sporadic in juvenile DM; however, abnormal findings assessed by electrocardiography (ECG) and echocardiography has been found both early $(3)$ and late $(4,5)$ in the disease course. Notably, even if the clinical relevance of these subtle cardiac abnormalities has yet to be determined, cardiac monitoring is recommended for juvenile DM patients (6).

Nailfold capillaroscopy (NFC) is a simple, non-invasive technique to evaluate the microvascular architecture. It has been hypothesised that NFC might be a putative biomarker in autoimmune rheumatic diseases; pilot studies in patients with systemic sclerosis (SSc) showed associations with peripheral vascular and pulmonary involvement (7). Additionally, microvascular changes of the nailfolds are associated with pulmonary involvement (7-9), and might be predictive for future severe organ involvement in SSc (9). SSc patients with striking microvascular abnormalities 
presented also more frequently cardiac involvement and decreased heart rate variability (HRV), although the findings are conflicting (7). In adult DM, NFC findings were associated with pulmonary, but not with cardiac, involvement $(10,11)$. Of note, cardiac related pathologies were rare in most of these studies $(7,10)$. Thus, there are data suggesting that NFC may be a useful tool to investigate organ involvement in adults with rheumatic diseases, however, there are no studies addressing this issue in juvenile DM or in other paediatric rheumatic diseases.

Our group has established a Norwegian juvenile DM cohort that was clinically examined after medium- to long-term disease duration. This cohort has been thoroughly described with regards to NFC (12), pulmonary (13) and cardiac involvement $(4,5,14)$. Patients presented more abnormal findings in all observed NFC measures than age- and sex-matched controls. Nailfold capillary density (NCD), low NCD (defined as $<6$ capillaries $/ \mathrm{mm}$ ) and neovascular pattern (defined as active or late scleroderma pattern) were shown to be the most applicable NFC measures (12). Compared with controls, patients had smaller lung volumes and reduced gas diffusion capacity; also, $37 \%$ of patients presented abnormalities assessed by highresolution computed tomography (HRCT) (13). Moreover, in our cohort, patients had cardiac abnormalities including decreased systolic (4) and diastolic function (5), reduced heart rate variability (HRV) (14) and more ECG pathologies (5), compared to the matched controls. No studies have investigated the association between NFC findings and pulmonary or cardiac involvement in juvenile DM. Thus, the objective of the present study was to investigate the possible relationship between NFC and pulmonary and cardiac measures in juvenile DM patients examined after medium- to long-term disease duration. 


\section{Patients and methods}

Study design and cohort. Our established Norwegian juvenile DM inception cohort consists of 60 patients diagnosed between January 1970 and June 2006 (15). Inclusion criteria were a probable or definitive diagnosis of dermatomyositis according to the Bohan and Peter criteria; disease onset before the age of 18 years, $\geq 24$ months from symptom onset to follow-up and age at follow-up $\geq 6$ years. Informed consent was obtained from all patients (and their parents, if age $<16$ years), and the Regional Ethics Committee approved the study (S-05144).

\section{Data collection and clinical and laboratory measurements. Patients were} clinically examined after mean 17-years disease duration. Disease onset was defined as the time of the first muscle or skin symptom, and disease duration as the time from disease onset to the follow-up. We have previously published data on NFC in relation to general disease variables in this cohort (12), also data on pulmonary (13) and cardiac outcomes $(4,5,14)$.

Nailfold capillaroscopy. A video microscope (Video Cap Video Microscope; Scalar Co. DS MediGroup, Milan, Italy) was used for NFC examinations (performed by HS) (12). The analyses were done by ZB who was blinded to clinical information. Videocap 8.20 software (Videocap; DS MediGroup, Milan, Italy) was used for image analysis. NCD and neovascular pattern (defined as active or late scleroderma pattern) were assessed as previously described in detail (12). The cut-off used for low NCD was $<6$ capillaries/mm (12). From our inception cohort, one patient was not examined with NFC, and one patient was excluded due to limited number of available NFC recordings of good quality; thus, data of 58 patients were used for further analyses. 


\section{Pulmonary assessment.}

PFT. All PFT measurements, including spirometry (forced vital capacity, FVC), measurements of gas diffusion (diffusing capacity for carbon monoxide, DLCO) and body plethysmography (total lung capacity, TLC), were performed on a computerised Vmax Pulmonary Function Unit (Viasys, Santa Ana, CA, USA). All spirometric variables were measured in accordance with the current guidelines (13). The PFT variables were expressed as percentage of predicted (13). Low FVC\%, TLC\% and DLCO\% were defined as less than the $5^{\text {th }}$ percentile of the predicted values and PFT abnormality was defined as low TLC\% and/or low DLCO\%.

HRCT. HRCT was performed in 56 patients using a LightSpeed 16 scanner (GE Healthcare, Milwaukee, WI, USA). An experienced radiologist read the images blinded to clinical information, and scored the presence of ILD (reticular pattern with or without traction bronchiectasis, and/or ground glass opacity), and airways diseases (bronchiectasis, and/or air trapping and/or micronodules) (13).

\section{Cardiac assessment.}

Echocardiography. Two-dimensional and Doppler echocardiography were performed and analysed blinded to patient information $(4,5)$. Diastolic function was measured by early diastolic tissue velocity (e') which was recorded in the mitral ring in two- and four-chambers views (5). Systolic function was measured by long axis strain (LAS) (mitral annulus displacement as percentage of end-diastolic left ventricular length) (4). A lower value of e' and LAS, suggests poorer diastolic or systolic function, respectively. 
Electrocardiography. A 12-channel ECG and a 24-hour ambulatory Holter monitoring were carried out as previously described in detail $(5,14)$. ECGs were analysed blinded to clinical information and classified as normal or pathological. The calculation of HRV (standard deviation of all normal-to-normal intervals corrected to the heart rate, CSDNN) was performed by software (Holtersoft Ultima v2.4.4, Novacor, Rueil-Malmaison, France) (14).

Statistical analysis. Differences were tested by the Student's $t$-test for continuous and normally distributed variables and by the Mann-Whitney $U$ test for continuous not-normally distributed variables, as appropriate. Chi-square was used to test differences between two groups for categorical variables. Correlations were determined by the Spearman's correlation coefficient $\left(r_{s}\right)$.

NCD is known to be dependent on age and possibly disease duration; therefore, multivariate logistic regression analysis was used to age adjust the associations between low NCD (dependent variable) and cardiac parameters as well as HRCT findings (age used as an independent variable). PFT variables are presented as percentage of predicted; thus, values were already corrected for age

To explore the possible effect of disease duration on the association between PFT variables and NCD and low NCD, respectively; multivariate linear and logistic regression analyses were performed with disease duration and various PFT variables as independent variables. Due to a strong intercorrelation between age and disease duration $(R=0.938 ; p<0.001)$ both variables could not be included as independent variables in the regression analyses.

Two-tailed tests were used for all calculations, and $p<0.05$ was considered significant; SPSS v.24 (SPSS, Chicago, Illinois, USA) was used for statistical 
analyses. Due to the explorative nature of the study, we did not adjust $p$-values for multiple comparisons.

\section{Results}

Patients' characteristics have previously been described in detail (15) and selected parameters are shown in Table 1 as background information. As previously described, 21/58 (36\%) of patients had low NCD (Table 1) and 24/58 (41\%) had neovascular pattern (12).

Table 1 also shows selected pulmonary data in juvenile DM patients, as well as in patients with normal and low NCD. Low NCD was associated with lower values of FVC\% and TLC\% (Figure 1); moreover, low NCD was associated with low DLCO\%. Signs of airways disease on HRCT were also more prevalent in the low NCD group.

There were weak to moderate correlations between NCD (as a continuous variable) and the following variables: FVC\% $\left(r_{s}=0.262, p=.047\right)$ and HRCT assessed airways disease $\left(r_{s}=-0.359, p=.007\right)$, but not with any other PFT or HRCT variables included in Table 1 (data not shown).

When adjusting the association between NCD / low NCD and all PFT variables (that are already age adjusted) for disease duration, FVC\% was no longer significantly associated with $N C D$ (Std B $0.219, \mathrm{p}=0.080$ ) but an association between TLC\% and NCD was found (Std B 0.295, $\mathrm{p}=0.018$ ). Both FVC\% and TLC\% remained associated with low NCD (OR 0.936 ( $p$ 0.18) and OR $0.931(p=0.006)$ ); while low DLCO lost significance (data not shown). Additionally, we analysed the differences in HRCT 
measures after adjusting for age; airways disease was more frequent in low NCD than in the normal NCD group.

Table 1 also shows selected cardiac data in juvenile DM patients, as well as in patients with normal and low NCD. We found no significant differences between patients with low and normal NCD, including ECG findings, HRV, and systolic or diastolic function assessed by echocardiography. Also, no significant associations between low NCD and cardiac measures were found after adjusting for age. No significant correlations between cardiac parameters and NCD (as a continuous variable) were found (data not shown).

Additionally, no associations between neovascular pattern and any pulmonary or cardiac variables were found (selected data are shown in Figure 2, the remaining data not shown).

\section{Discussion}

To our knowledge, this is the first study to investigate the relationship between NFC findings and pulmonary and cardiac involvement in juvenile DM patients. We found associations between NFC variables and lung involvement; low NCD was associated with smaller lung volumes, reduced gas diffusion capacity and HRCT detected airways disease. No significant associations between NFC and cardiac involvement were detected.

The representativeness of our juvenile DM cohort has been described earlier (15); we believe it covers the vast majority of juvenile DM patients diagnosed from 1970 to 2006 in Norway. 
Our key finding is the relationship between low NCD and smaller lung volumes (FVC and TLC) and reduced gas diffusion (low DLCO) in juvenile DM. In SSc, a recent study has shown consistent results with our findings (8) when comparing patients with low (<7 capillaries/mm) and normal NCD: both FVC\% (87 vs 101\%) and DLCO\% (71 vs $86 \%$ ) were decreased in patients with low NCD. This is comparable to our results, where $\mathrm{FVC} \%$ was decreased (90 vs $99 \%$ ) and DLCO\% was borderline decreased (77 vs $84 \%)$ in patients with low vs normal NCD.

HRCT assessed airways disease was more prevalent in patients with low NCD, than in patients with normal NCD. There was no significant difference in the prevalence of HRCT detected ILD between the groups. In SSc, numerous studies have shown an association between NFC measures and lung involvement (9); reduced NCD was associated with ILD (8) and SSc patients with lung fibrosis presented decreased NCD and more bushy capillaries as compared with patients with idiopathic pulmonary fibrosis (9). Thus, even if a few SSc studies did not find association between capillaroscopic variables and lung involvement (9), microvascular changes seem to reflect pulmonary involvement in SSc $(7,8)$. In our cohort, although decreased lung volumes and higher incidence of HRCT detected airways disease were found in patients with low NCD, a considerable proportion of patients with normal NCD also presented lung involvement. Thus, even if microvascular involvement seems to be relevant in the development of pulmonary manifestations, other factors are likely to contribute to the process in juvenile DM.

No significant association was revealed between low NCD and cardiac involvement. None of the NFC parameters correlated significantly with systolic or diastolic function; neither with ECG pathologies nor HRV. Although clinically relevant cardiac disease is rare in idiopathic inflammatory myopathies including juvenile DM, we have previously 
shown that subclinical cardiac involvement is present in approximately $1 / 4$ of the patients with juvenile DM $(4,5,14)$. The exact mechanism underlying cardiac involvement is unknown, but atherosclerosis, small vessel vasculopathy and myocardial as well as systemic inflammation may play a role in the process (16). Since we did not find any significant association between NCD and parameters of cardiac dysfunction, our data do not support that vasculopathy is an important underlying mechanism for cardiac involvement in juvenile DM. However, our study is limited by a small sample size, which makes it challenging to study rare outcomes; thus we might be underpowered to demonstrate associations between cardiac involvement and NFC.

There is a known relationship between age and NCD (17) and age and possible disease duration might influence the associations between pulmonary and cardiac variables and NCD. In our study, age and disease duration were strongly intercorrelated $(r=0.938)$. Adjusting for these factors did not substantially influence the results; we found robust associations between lung volumes and NCD. We have previously studied NCF findings in the same cohort and compared findings with age and sex matched controls. Notably, the correlations between NCD and age were comparable in patients $(R=0.407 ; p=0.002)$ and controls $(R=0.431 ; p=0.003)$ (12), which supports that this association is mainly an effect of aging and not an effect of disease duration.

In conclusion, juvenile DM patients with low NCD have impaired PFT, and more frequent HRCT abnormalities compared to controls. In contrast, we did not find significant associations between capillaroscopic variables and cardiac involvement. Our results suggest that systemic microvascular remodeling might be an underlying mechanism for the pulmonary involvement in juvenile DM. However, further and 
preferably larger studies are needed to examine NCD as a potential biomarker for organ involvement.

\section{Author contributions}

Study conception and design. Sanner, Barth and Sjaastad

Acquisition of data. Aaløkken, Sanner and Sjaastad

Analysis and/or interpretation of data. All authors

Drafting of manuscript. Barth, Sanner and Sjaastad

Critical review of manuscript and final approval of submission. All authors

\section{REFERENCES}

1. Lowry CA, Pilkington CA. Juvenile dermatomyositis: extramuscular manifestations and their management. Curr Opin Rheumatol. 2009;21(6):575-80.

2. Kobayashi N, Takezaki S, Kobayashi I, Iwata N, Mori M, Nagai K, et al. Clinical and laboratory features of fatal rapidly progressive interstitial lung disease associated with juvenile dermatomyositis. Rheumatology. 2015;54(5):784-91.

3. Cantez S, Gross GJ, MacLusky I, Feldman BM. Cardiac findings in children with juvenile Dermatomyositis at disease presentation. Pediatric rheumatology online journal. 2017;15(1):54.

4. Schwartz T, Sanner H, Gjesdal O, Flato B, Sjaastad I. In juvenile dermatomyositis, cardiac systolic dysfunction is present after long-term follow-up and is predicted by sustained early skin activity. Annals of the rheumatic diseases. 2014;73(10):1805-10.

5. Schwartz T, Sanner H, Husebye T, Flato B, Sjaastad I. Cardiac dysfunction in juvenile dermatomyositis: a case-control study. Annals of the rheumatic diseases. 2011;70(5):766-71.

6. Enders FB, Bader-Meunier B, Baildam E, Constantin T, Dolezalova P, Feldman BM, et al. Consensus-based recommendations for the management of juvenile dermatomyositis. Annals of the rheumatic diseases. 2017;76(2):329-40.

7. Smith V, Decuman S, Sulli A, Bonroy C, Piettte Y, Deschepper E, et al. Do worsening scleroderma capillaroscopic patterns predict future severe organ involvement? a pilot study. Annals of the rheumatic diseases. 2012;71(10):1636-9.

8. Castellvi I, Simeon-Aznar CP, Sarmiento M, Fortuna A, Mayos M, Geli C, et al. Association between nailfold capillaroscopy findings and pulmonary function tests in patients with systemic sclerosis. The Journal of rheumatology. 2015;42(2):222-7.

9. Soulaidopoulos S, Triantafyllidou E, Garyfallos A, Kitas GD, Dimitroulas T. The role of nailfold capillaroscopy in the assessment of internal organ involvement in systemic sclerosis: A critical review. Autoimmun Rev. 2017;16(8):787-95.

10. Mugii N, Hasegawa M, Matsushita T, Hamaguchi Y, Horie S, Yahata T, et al. Association between nail-fold capillary findings and disease activity in dermatomyositis. Rheumatology.

2011;50(6):1091-8. 
11. Selva-O'Callaghan A, Fonollosa-Pla V, Trallero-Araguas E, Martinez-Gomez X, Simeon-Aznar $\mathrm{CP}$, Labrador-Horrillo $\mathrm{M}$, et al. Nailfold capillary microscopy in adults with inflammatory myopathy. Semin Arthritis Rheum. 2010;39(5):398-404.

12. Barth Z, Witczak BN, Flato B, Koller A, Sjaastad I, Sanner H. Assessment of Microvascular Abnormalities by Nailfold Capillaroscopy in Juvenile Dermatomyositis After Medium- to Long-Term Followup. Arthritis care \& research. 2018;70(5):768-76.

13. Sanner H, Aalokken TM, Gran JT, Sjaastad I, Johansen B, Flato B. Pulmonary outcome in juvenile dermatomyositis: a case-control study. Annals of the rheumatic diseases. 2011;70(1):86-91. 14. Barth Z, Nomeland Witczak B, Schwartz T, Gjesdal K, Flato B, Koller A, et al. In juvenile dermatomyositis, heart rate variability is reduced, and associated with both cardiac dysfunction and markers of inflammation: a cross-sectional study median 13.5 years after symptom onset.

Rheumatology. 2016;55(3):535-43.

15. Sanner H, Gran JT, Sjaastad I, Flato B. Cumulative organ damage and prognostic factors in juvenile dermatomyositis: a cross-sectional study median 16.8 years after symptom onset.

Rheumatology. 2009;48(12):1541-7.

16. Schwartz T, Diederichsen LP, Lundberg IE, Sjaastad I, Sanner H. Cardiac involvement in adult and juvenile idiopathic inflammatory myopathies. RMD Open. 2016;2(2):e000291.

17. Dolezalova P, Young SP, Bacon PA, Southwood TR. Nailfold capillary microscopy in healthy children and in childhood rheumatic diseases: a prospective single blind observational study. Annals of the rheumatic diseases. 2003;62(5):444-9. 